\title{
Do Professional Development Programs for Maintenance of Certification (MOC) Affect Quality of Patient Care?
}

\author{
James M. Galliher, PhD, Brian K. Manning, MPH, Stephen M. Petterson, PhD, \\ L. Miriam Dickinson, PhD, Elias C. Brandt, BS, Elizabeth W. Staton, MSTC \\ Robert L. Phillips, MD, MSPH, and Wilson D. Pace, MD
}

Objective: The objective of this study was to examine the relationship between physicians' completion of American Board of Family Medicine (ABFM) Maintenance of Certification (MOC) modules and the quality of medical care delivered.

Methods: Physicians from the Electronic National Quality Improvement and Research Network (eNQUIRENet) were enrolled. Data from their electronic health records were compared before and after they completed one or more MOC modules for family physicians (Self-Assessment Module [Part II MOC] and Performance in Practice Module [Part IV MOC]; SAM/PPM). Process data and other quantitative clinical measures for all adult patients with a diagnosis of type 2 diabetes were gathered from each study physician. General linear mixed effects models were used to analyze data before and after the MOC modules, adjusting for clustering of patients within physicians.

Results: Physicians participating in SAM/PPM activities demonstrated greater improvements over time in 11 of 24 measures in process and intermediate outcome measures related to type 2 diabetes care compared with non-SAM/PPM participants. All groups demonstrated improvements over time.

Conclusion: Participation in SAM/PPM activities is associated with greater improvements in care, but the association between activity undertaken and specific improvements is difficult to demonstrate.( $\mathrm{J}$ Am Board Fam Med 2014;27:19-25.)

Keywords: Certification, Diabetes Mellitus, Quality of Health Care, Training

National standards for certification of physicians resulted in the creation of the Advisory Board for Medical Specialties in 1933. This advisory board eventually became the American Board of Medical Specialties, which currently supports 24 different medical specialty board organizations. All are fo-

This article was externally peer reviewed.

Submitted 29 March 2013; revised 7 August 2013; accepted 12 August 2013.

From the American Academy of Family Physicians, Leawood, KS (JMG, BKM, LMD, ECB, EWS, WDP); the Robert Graham Center, Washington, DC (SMP); and the American Board of Family Medicine, Lexington, KY (RLP)

Funding: This project was a collaboration of the American Academy of Family Physicians National Research Network (AAFP NRN), the Robert Graham Center, and the American Board of Family Medicine (ABFM), with funding provided by the ABFM.

Conflict of interest: none declared.

Corresponding author: Brian K. Manning, MPH, AAFP National Research Network, 11400 Tomahawk Creek Parkway, Leawood, KS 66211-2680 (E-mail: bmanning@aafp.org). cused on assessing the ability of licensed physicians to competently practice in specific domains. Certification "signals a board certified physician's commitment and expertise in consistently achieving superior clinical outcomes." While this is an appropriate goal, there is little empirical evidence that board certification or the new Maintenance of Certification (MOC) process assures this. ${ }^{2}$

The American Board of Family Medicine (ABFM) was the first medical board to require recertification for its members. It has focused on competency-based assessments. The ABFM has embraced the new concepts of MOC and a more continuous process of care assessment and improvement. We sought to examine the relationship (if any) between physicians' completion of ABFM MOC modules and the quality of medical care delivered as measured from electronic health records (EHRs). 
This retrospective pilot study investigated the ability to demonstrate an effect of 2 specific MOC modules for family physicians-the Self-Assessment Module (SAM) from Part II MOC and the Performance in Practice Module (PPM) from Part IV MOC - on the quality of care delivered by family physicians to their patients. The study focused on study physicians' patients with an established diagnosis of type 2 diabetes mellitus. In so doing, we also assessed the feasibility of obtaining and using these electronic data to investigate such questions and highlighted issues needing attention in larger systematic investigations.

\section{Methods}

This study used the Electronic National Quality Improvement and Research Network (eNQUIRENet), a subnetwork of the American Academy of Family Physicians (AAFP) National Research Network, previously named the Distributed Ambulatory Research in Therapeutics Network (DARTNet). eNQUIRENet is a network of medical organizations currently representing more than 2500 clinicians (45\% family physicians) and 3 million patients. EHR data from geographically and organizationally separate databases are linked in a manner such that one query may access separate databases and return results while conforming to individual organization's privacy and confidentiality standards. ${ }^{3,4}$

\section{Recruitment of Study Physicians}

Family physicians were recruited from eNQUIRENet. At the start of the project in March 2010, the network included 28 organizations with more than 1500 clinicians (approximately 60\% family physicians) and more than 1000,000 patients. ${ }^{5}$ Our goal was to recruit 90 to 120 physicians, or 30 to 40 doctors per each of 3 study groups (described below).

Physicians were recruited from 23 of 28 eNQUIRENet organizations; 5 organizations had administrative or technical reasons precluding their participation. Recruitment occurred through a single contact at each organization. Written informed consent was required of physicians agreeing to participate. We clarified with each organization's contact person how best to invite their family physicians into the study. Some organizations allowed project staff to directly contact their physicians by mail, E-mail, or phone, whereas others made these contacts themselves by informing their physicians about this research opportunity. Physician recruitment occurred June 17 through August 31, 2010.

\section{Matching DARTNet Study Physicians to the ABFM Database}

The ABFM MOC database identifies the topic and completion date of each SAM or PPM completed by ABFM diplomates. Using the first and last names of these diplomates, we matched the ABFM MOC database to the study physicians. Differences in the spelling of names or name changes were hand-edited to obtain a $100 \%$ match.

\section{Obtaining SAM/PPM Completion Data from the ABFM Database}

The information related to ABFM SAM and/or PPM participation was used to classify each study doctor into 1 or 3 mutually exclusive study groups:

1. Physicians completing a SAM and/or PPM in diabetes from January 1, 2007, to December 31, 2009 (at least some of these physicians also completed a SAM and/or PPM in a topic other than diabetes during this same period);

2. Physicians completing a SAM and/or PPM in a topic other than diabetes during 2007 to 2009; and

3. Physicians not completing either a SAM or PPM; these physicians' ABFM records reported no completion of a SAM/PPM during this period.

For physicians with $\geq 2$ SAMs/PPMs in diabetes (group 1) or some other topic (group 2), their program completion date reflected the most recent module completed. Physicians not completing a $\mathrm{SAM} / \mathrm{PPM}$ during this period were assigned the median date for the diabetes cohort (October 2, 2008) for purposes of EHR data draws and analyses. We used the completion date of doctors' most recent SAM or PPM (groups 1 and 2) as the last date of the period before the SAM/PPM. The completion date established the periods before and after the modules for each physician. To illustrate using a doctor's completion date of July 13, 2009:

1. Date for start of period before the modules: July 14, 2008.

2. Date for end of period before the modules: July 13, 2009. 
Table 1. Clinical Process and Intermediate Patient Outcomes for the Before and After Periods Obtained for Patients of DARTNet Study Physicians

\begin{tabular}{|c|c|}
\hline Individual Clinical Measures & $\begin{array}{l}\text { Outcome } \\
\text { Description }\end{array}$ \\
\hline \multicolumn{2}{|l|}{ 1. Hemoglobin A1C } \\
\hline 1A. Process outcome (date) & Every 12 months \\
\hline 1B. Intermediate patient outcome (level) & $<8 \%$ \\
\hline \multicolumn{2}{|l|}{ 2. Blood pressure (systolic and diastolic) } \\
\hline 2A. Process outcome (date) & Every 12 months \\
\hline 2B. Intermediate patient outcome (level) & $\begin{array}{l}\text { Systolic }<140 \\
\quad \text { diastolic }<90\end{array}$ \\
\hline \multicolumn{2}{|l|}{ 3. Low-density lipoprotein cholesterol } \\
\hline 3A. Process outcome (date) & Every 12 months \\
\hline 3B. Intermediate patient outcome (level) & $<100 \mathrm{mg} / \mathrm{dL}$ \\
\hline \multicolumn{2}{|l|}{ 4. Protein-to-creatinine ratio } \\
\hline 4A. Process outcome (date) & Every 12 months \\
\hline 4B. Intermediate patient outcome (level) & $<30 \mathrm{mg} / \mathrm{mmol}$ \\
\hline \multicolumn{2}{|l|}{$\begin{array}{l}\text { 5. Angiotensin-converting enzyme and } \\
\text { angiotensin receptor blocker }\end{array}$} \\
\hline 5A. Process outcome (date) & Every 12 months \\
\hline 5B. Intermediate patient outcome (date) & Every 12 months \\
\hline \multicolumn{2}{|l|}{ 6. Albumin-to-creatinine ratio } \\
\hline 6A. Process outcome (date) & Every 12 months \\
\hline 6B. Intermediate patient outcome (date) & $<30 \mathrm{mg} / \mathrm{dL}$ \\
\hline
\end{tabular}

3. Date for start of period after the modules: July 14, 2009.

4. Date for end of period after the modules: July $13,2010$.

\section{Identification of Applicable Study Patients}

Each patient within a study physician's practice who was (1) at least 18 years of age at the start of their study doctor's period before the module and (2) had a diagnosis of type 2 diabetes before the start of their study doctor's period before the module was included in the analysis.

\section{Identification and Abstraction of Study Patient Data}

The clinical data obtained for this study are shown in Table 1. Patients were attributed to study physicians either through the EHR or using an algorithm based on either a plurality of visits during the study period or on their initial visit. All patients used for this analysis were required to have at least one visit during the period after the intervention. A given study patient may also have seen another doctor during the study period.

\section{Developing Guideline Concordance Measures}

Quality of care delivered by physicians to patients with type 2 diabetes mellitus was tied to clinical guidelines recommended by the American Diabetes Association. ${ }^{6}$ Quantitative outcome measures were converted to dichotomous outcomes as being either concordant or not concordant with recommended clinical guidelines. These measures were converted to process measures (eg, dates on which a given test/examination was performed relative to clinical recommendations) unrelated to the actual quantitative clinical measure. Dichotomous clinical measures (angiotensin-converting enzyme $[\mathrm{ACE}]$ /angiotensin receptor blocker $[\mathrm{ARB}]$ usage and foot examination) were treated as both process and intermediate patient outcomes (yes/no).

Individual measures were combined into $2 \mathrm{com}-$ posite concordance measures: (1) process outcome concordance, based on the performance of an activity, and (2) intermediate patient-outcome concordance, based on the actual clinical measure except when the process was also an outcome, such as performing a foot examination.

The values for each composite concordance measure varied from 0 (no patient guideline concordance across the 6 individual items) to 6.0 (complete patient guideline concordance). When expressed as proportions, these values varied from 0.0 (0 of 6 items concordant) to 1.0 (6 of 6 items concordant).

\section{Statistical Analysis}

Descriptive statistics (means, standard deviations, proportions, frequency distributions) were generated for patient sociodemographic measures, diabetes process of care, and clinical outcomes. General (generalized) linear mixed effects regression models, adjusting for patient age and sex, were used to examine differences in outcomes over time by group. Patient and physician random effects were included to adjust for clustering of observations within patients and clustering of patients within physicians. For each patient-level outcome, regression models included an indicator variable for (1) period before or after completion of the module, (2) physician group (the 3 groups of study doctors described earlier), and (3) an interaction term between the period and physician group. Although there was insufficient variability to include practice as a random effects, simulation studies with a 3-level data structure indicate less bias in param- 
Table 2. DARTNet Physician Study Groups and Eligible Patients at Before and at After Completion of SAM/PPM Periods

\begin{tabular}{lcccr}
\hline & & \multicolumn{3}{c}{ Patients } \\
\cline { 3 - 5 } Study Group & \begin{tabular}{c} 
Doctors \\
\cline { 3 - 5 }
\end{tabular} & No. & Mean (SD) & Range \\
\hline Diabetes SAM/PPM* & 17 & 1169 & $68.7(53.2)$ & $1-163$ \\
Nondiabetes SAM/PPM & 20 & 1345 & $67.2(79.5)$ & $5-364$ \\
No SAM or PPM & 39 & 2163 & $55.5(50.0)$ & $11-196$ \\
Totals & 76 & 4677 & $61.5(59.3)$ & $1-364$
\end{tabular}

*There were an additional 3 doctors in this group who were excluded from analysis because of a lack of any eligible patients. ${ }^{\dagger}$ There were an additional 7 doctors in this group who were excluded from analysis because of a lack of any eligible patients. SAM/PPM, Self-assessment Module/Performance in Practice Module.

eter estimates using a 2-level model ignoring the third level than for a 2-level model ignoring the second level. ${ }^{7}$ Thus we elected to include physician rather than practice as a random effect. In addition, these approaches can accommodate some singletons and small (ie, $<5$ patients) lower-level sample sizes $^{8}$ if the number of small samples is small relative to the total number of upper-level units. In this study there were only 2 physicians with fewer than 5 patients. All estimates are shown at the means for age and sex (average age, 61 years; 52\% female). This project was approved by the AAFP Institutional Review Board.

\section{Results}

\section{Recruitment Results}

During the 10-week recruiting period, 86 family physicians from 16 practices enrolled (mean, 5.4; median, 2.5; range, 1-19 doctors per practice). We are not able to estimate a response rate since we do not know the number of family physicians actually invited to participate. Although 86 physicians enrolled, $10(12 \%)$ were new to their practice and did not have patients who met the eligibility criteria related to timing of patient visits. Our final numbers were 76 doctors from 15 eNQUIRENet practices.

Doctors and their eligible patients by study group are shown in Table 2. Both SAM/PPM groups had roughly the same number of doctors (17 and 20, or 49\%), while the other 39 doctors (51\%) had neither a SAM nor PPM. Table 2 also shows the number of eligible patients for analysis $(\mathrm{n}=4677)$. The differences in the average number of patients per physician cohort (55.5-68.7) was not statistically significant $(P=.66)$; however, the standard deviation within each cohort was quite large (Table 2).

Tables 3 and 4 show before and after estimates for process of care, intermediate outcomes, and composite outcomes. When physicians from the type 2 diabetes mellitus SAM/PPM group were compared with those with no SAM/PPM, the former showed statistically greater improvement from

Table 3. Process Outcomes

\begin{tabular}{|c|c|c|c|c|c|c|c|c|}
\hline Group & Time & $\begin{array}{l}\text { Foot } \\
\text { Exam }\end{array}$ & $\mathrm{ACE}$ & $\begin{array}{l}\text { Hemoglobin } \\
\text { A1C Check }\end{array}$ & $\begin{array}{c}\mathrm{BP} \\
\text { check }\end{array}$ & $\begin{array}{l}\text { LDL } \\
\text { Check }\end{array}$ & $\begin{array}{l}\text { Albumin-to- } \\
\text { Creatinine Ratio Check }\end{array}$ & $\begin{array}{c}\text { Comp LP* } \\
\text { (Average Score) }\end{array}$ \\
\hline \multirow[t]{2}{*}{ 1. Diabetes } & Before & 9.20 & 15.10 & 49.44 & 61.30 & 49.40 & 12.97 & 1.99 \\
\hline & After & 19.40 & 24.90 & 70.70 & 84.80 & 68.50 & 25.97 & 2.96 \\
\hline Absolute difference & & 10.20 & 9.80 & 21.26 & 23.50 & 19.10 & 13.00 & 0.97 \\
\hline$P$ value $^{\dagger}$ & & .0024 & .0003 & .9757 & $<.0001$ & .9215 & .8238 & .1713 \\
\hline \multirow[t]{2}{*}{ 2. Other } & Before & 4.90 & 24.80 & 38.90 & 58.30 & 37.10 & 16.11 & 1.80 \\
\hline & After & 14.70 & 18.10 & 73.80 & 84.20 & 72.80 & 37.82 & 3.02 \\
\hline Absolute difference & & 9.80 & -6.70 & 34.90 & 25.90 & 35.70 & 21.71 & 1.22 \\
\hline$P$ value $^{\ddagger}$ & & .0007 & $<.0001$ & $<.0001$ & $<.0001$ & $<.0001$ & $<.0001$ & $<.0001$ \\
\hline \multirow[t]{2}{*}{ 3. None } & Before & 3.70 & 19.30 & 58.40 & 65.70 & 51.20 & 18.90 & 2.18 \\
\hline & After & 18.70 & 21.40 & 79.70 & 82.60 & 70.50 & 32.31 & 3.06 \\
\hline Absolute difference & & 15.00 & 2.10 & 21.30 & 16.90 & 19.30 & 13.41 & 0.88 \\
\hline
\end{tabular}

Data are percentages unless otherwise indicated.

${ }^{*}$ Comp LP is the average of the sum of all measures at the patient level (possible score of $0-6$ ).

${ }^{\dagger}$ For diabetes (group 1) vs. none (group 3).

${ }^{\ddagger}$ For other (group 2) vs. none (group 3).

ACE, angiotensin-converting enzyme; BP, blood pressure; LDL, low-density lipoprotein. 
Table 4. Intermediate Outcomes

\begin{tabular}{|c|c|c|c|c|c|c|}
\hline Group & Time & $\begin{array}{l}\text { Hemoglobin } \\
\text { A1C }\end{array}$ & $\mathrm{BP}$ & LDL & $\begin{array}{l}\text { Albumin-to- } \\
\text { Creatinine Ratio }\end{array}$ & $\begin{array}{c}\text { Comp LI* } \\
\text { (Average Score) }\end{array}$ \\
\hline \multirow[t]{2}{*}{ 1. Diabetes } & Before & 22.5 & 54.7 & 33.7 & 10.2 & 1.46 \\
\hline & After & 33.2 & 76.6 & 50.8 & 20.5 & 2.26 \\
\hline Absolute difference & & 10.5 & 21.9 & 17.1 & 10.3 & 0.80 \\
\hline$P$ value $^{\dagger}$ & & .3417 & $<.0001$ & .1702 & .7982 & .0048 \\
\hline \multirow[t]{2}{*}{ 2. Other } & Before & 20.7 & 49.3 & 26.0 & 11.3 & 1.37 \\
\hline & After & 34.3 & 74.0 & 47.9 & 27.4 & 2.17 \\
\hline Absolute difference & & 13.6 & 24.7 & 21.9 & 16.1 & 0.79 \\
\hline$P$ value $^{\ddagger}$ & & .5043 & $<.0001$ & .0001 & .0008 & .0504 \\
\hline \multirow[t]{2}{*}{ 3. None } & Before & 29.1 & 57.0 & 32.8 & 13.8 & 1.56 \\
\hline & After & 41.5 & 71.3 & 47.1 & 24.5 & 2.25 \\
\hline Absolute difference & & 12.4 & 14.3 & 14.3 & 10.7 & 0.69 \\
\hline
\end{tabular}

Data are percentages unless otherwise indicated.

${ }^{*}$ Comp LI is the average of the sum of all measures at the patient level (possible score of $0-4$ ).

${ }^{\dagger}$ Diabetes (group 1) vs. none (group 3).

${ }^{\ddagger}$ Other (group 2) vs. none (group 3).

$\mathrm{BP}$, blood pressure; LDL, low-density lipoprotein.

the period before to the period after the program $(P<.01)$ for ACE/ARB use, blood pressure $(\mathrm{BP})$ checks, and BP control. The type 2 diabetes mellitus group demonstrated smaller improvement for performance of foot exams, although the final absolute percentage of concordance was not different. The composite intermediate outcome measure indicated significantly greater improvement in the SAM/PPM group as well $(P=.048)$.

In either process or outcome variables, when physicians from the other SAM/PPM group were compared with those with no SAM/PPM, the former showed statistically greater improvement $(P<$ .01) in ACE/ARB use, BP checks, low-density lipoprotein (LDL) checks, albumin-to-creatinine ratio checks, and the composite process of care score. The other SAM/PPM group demonstrated greater process improvement for intermediate outcomes for BP, LDL, and microalbuminuria. These physicians showed less improvement in foot exams and an actual decline in ACE/ARB use.

Overall, of the 14 statistically significant comparisons in both measurement approaches, 11 showed greater improvements in patient concordance from the period before to the period after the module for one of the SAM/PPM groups compared with the control group with no SAM/PPM program completion (see Tables 3 and 4). Sensitivity analyses varying the patient attribution approach and varying the mixed effects models were conducted, with similar results (data not shown), al- though some measures lost significance when corrected for multiple comparisons. Overall, all groups improved over time in most measures.

\section{Discussion}

To our knowledge, this is the first study to systematically investigate the associated effect of 2 specific professional development programs required for MOC_namely, SAM from Part II MOC and PPM from Part IV MOC - on the quality of care delivered by family physicians. The results support the concept of some impact on some aspects of diabetes care quality as a result of the MOC process, although this improvement seems to occur on a backdrop of temporal trends for improved care. There was considerable variation in specific concordance measures, whether process or intermediate outcomes, from a low of $3.5 \%$ of foot examination concordant at baseline in the no SAM/PPM group to a high of $97.4 \%$ of patients concordant for BP measurement in the period after testing. The BP finding is likely to be inflated by the fact that patients were required to have at least one visit in the period after intervention and BP is checked at virtually all primary care visits.

The majority of the significant between-group differences based on change in underlying performance before and after the intervention favored the groups that had taken one or more SAMs or completed a PPM. The finding that the group without 
a type 2 diabetes mellitus SAM/PPM improved on more diabetes measures than the group with a type 2 diabetes mellitus SAM/PPM is interesting. One interpretation could be a lack of effect of the SAM/ PPM process. Another possible interpretation is that many of the measures of interest appear in other SAM/PPM activities; for instance, LDL and $\mathrm{BP}$ control are components of the cardiovascular SAM, and use of ACE inhibitors in patients with diabetes is a component of the hypertension SAM. Thus, it is likely that there could be an interaction of effects across MOC activities for these types of measures. If this is the case, these should be seen as positive phenomena since overall patient health is the final goal of MOC activities.

There is a concern among some continuing medical education providers that physicians attend continuing medical education activities in areas they are already interested in and better at. Thus, continuing medical education may have lesser impact on the lowest performing providers. In this project the patients in the type 2 diabetes mellitus SAM/PPM group started higher in 5 of 6 measures compared with the group with no SAM/PPM on clinical concordance (process and intermediate patient outcomes). These findings support the concern that physicians tend to take continuing medical education in areas where they are already relatively better providers of care.

\section{Study Limitations}

This pilot study has a number of limitations.

\section{Number of Study Doctors}

First, we had a limited number of family physicians responding to our invitation for the study, and thus we did not meet our initial goal of at least 30 doctors per each of 3 study groups. The reduced number of physicians per the 2 SAM/PPM study groups may have hindered our ability to find statistical significant differences because of reduced power. Physicians may continue to be wary of having their care examined at this level, even though no physician-identifying data were provided either to the ABFM or to a physician's clinical organization. Because we had to approach each eNQUIRENet organization contact to recruit individual physicians, we have no way of knowing the number of potentially eligible family physicians that actually received a study invitation. Thus we do not know the response rate or how representative the study doctors (and their practice patterns) compare with the overall population of eNQUIRENet physicians. Physician participation required signing a short consent form. Physicians were paid a nominal amount for their time to review the consent form. Thus physician burden for entry into this study was low.

\section{Generalizability of Study Results}

Another limitation is related to the generalizability of study results. As is the case with most practicebased network studies, the doctors self-selected into the study. Thus we do not know how representative the study doctors (and their practice patterns) are compared to the overall population of US family physicians. Previous evaluations of differences between AAFP National Research Network physicians' self-reported practice patterns and randomly selected AAFP members have not demonstrated differences, ${ }^{9}$ although physicians in eQUIRENet were not included in those analyses, limiting extension to this study.

\section{Assignment of Eligible Patients to Study Doctors}

Another limitation to any analysis of physician performance relates to attribution strategies. Some, but not all, clinical organizations link patients and clinicians in their EHR. Even when assignment is clear in the EHR, there is generally no date attached to this assignment, and thus the accuracy of the data are not clear. Furthermore, an analysis of patient-provider visit frequency often does not align with the EHR assignment of the physician of record. Another approach to attribution for research purposes would be to have all practice doctors who had seen the patient during the study period assigned to a given patient. This overlapping of patients between cohorts would markedly complicate analysis and was not considered for this study. A common approach is to invoke the "plurality of patient visits" rule. Patients are assigned to the physician who saw the patients during the plurality of that patient's visits during the study period. Patient assignment when $\geq 2$ physicians are seen the same number of times can be handled in 3 ways: random assignment, assignment to the earliest physician, or assignment to the last physician seen. We studied 2 of these models: earliest physician seen and analysis limited to patients who saw the same physician in both study periods. These approaches had minimal effects on the results. 
It is important to recognize that the results were analyzed at the physician level, and physicians in 2 or 3 of the MOC groups often existed in the same clinical organization. Thus a difference in crossorganizational quality improvement activities cannot explain the findings because physicians in all groups may have been exposed to the same quality improvement activities. These organizational activities may explain the overall improvement trends.

\section{Conclusion}

Despite these and other possible limitations, we found that it is quite feasible to (1) successfully match physicians with their ABFM records of program completion; (2) categorize these doctors into 3 mutually exclusive study groups; (3) identify and use multiple methods for assigning eligible patients to each study doctor (although we reported only one here); (4) obtain the needed patient clinical data from disparate EHRs; and (5) design, program, and describe the composite process and intermediate outcome concordance measures. The results support the concept that participation in MOC activities is associated with improvements of care, although the ability to track impact based on the particular MOC activities seems limited, at least in clinical areas with significant overlap of measurements.

The authors thank the eNQUIRENet health care organizations and physicians who agreed to participate in this investigation.

\section{References}

1. American Board of Medical Specialties. What board certification means. Available from: http://www.abms. org/about_board_certification/means.aspx. Accessed January 9, 2013.

2. Iglehart JK, Baron RB. Ensuring physicians' competence-is maintenance of certification the answer? N Engl J Med. 2012;367:2543-9.

3. Libby AM, Pace W, Bryan C, et al. Comparative effectiveness research in DARTNet primary care practices: point of care data collection on hypoglycemia and over-the-counter and herbal use among patients diagnosed with diabetes. Med Care. 2010; 48(6 Suppl):S39-44.

4. Parnes B, Fernald D, Coombs L, et al. Improving the management of skin and soft tissue infections in primary care: a report from State Networks of Colorado Ambulatory Practices and Partners (SNOCAP-USA) and the Distributed Ambulatory Research in Therapeutics Network (DARTNet). J Am Board Fam Med. 2011;24:534-42.

5. Pace WD, Cifuentes M, Valuck RJ, Staton EW, Brandt EC, West DR. An electronic practice-based network for observational comparative effectiveness research. Ann Intern Med. 2009;151:338-40.

6. Standards of medical care in diabetes-2010. Diabetes Care. 2010;33(Suppl 1):S11-61.

7. Ten Have TR, Kunselman AR, Tran L. A comparison of mixed effects logistic regression models for binary response data with two nested levels of clustering. Stat Med. 1999;18:947-60.

8. Littell RC, Milliken GA, Stroup WW, Wolfinger RD. SAS system for mixed models. 2nd ed. Cary, NC: SAS Institute, Inc. 1996.

9. Galliher JM, Bonham AJ, Dickinson LM, Staton EW, Pace WD. Representativeness of PBRN physician practice patterns and related beliefs: the case of the AAFP National Research Network. Ann Fam Med. 2009;7:547-54. 